\title{
Construyendo mejores sociedades: los grupos escolares y el proceso de inclusión-exclusión educativa
}

Building better societies: scholar groups and educational inclusion-exclusion process

\author{
Volumen 19, Número 1 \\ Enero-Abril \\ pp. 1-18
}

\section{Este número se publica el 1 de enero de 2019}

DOI: 10.15517/aie.v19i1.35471

\author{
Patricia Batista Sardain \\ Jorge Enrique Torralbas Oslé
}

Revista indizada en REDALYC, SCIELO

Revista distribuida en las bases de datos:

LATINDEX, DOAJ, REDIB, IRESIE, CLASE, DIALNET, SHERPA/ROMEO, QUALIS-CAPES, MIAR

Revista registrada en los directorios:

ULRICH'S, REDIE, RINACE, OEI, MAESTROTECA, PREAL, $\underline{\text { CLACSO }}$ 


\title{
Construyendo mejores sociedades: los grupos escolares y el proceso de inclusión-exclusión educativa \\ Building better societies: scholar groups and educational inclusion-exclusion process
}

\author{
Patricia Batista Sardain ${ }^{1}$ \\ Jorge Enrique Torralbas Oslé
}

\begin{abstract}
Resumen: La escuela tiene el reto de ser el espacio donde se decide el rumbo de nuestras sociedades. En el empeño de sociedades más justas e inclusivas es preciso comprender el entramado socioeducativo y su impacto de forma más compleja, especialmente el grupo escolar como espacio de mediación. Para ello se propone, como alternativa teórica para el análisis de los procesos de inclusión-exclusión educativa, un marco conceptual que comprende las desigualdades desde la subjetividad social/grupal y coloca la mirada sobre la responsabilidad institucional. En este ensayo discutiremos sobre la responsabilidad de las instituciones escolares en las garantías para la inclusión, el papel del personal docente en el diseño de la actividad escolar y el funcionamiento de los grupos escolares desde su relación con el proceso de inclusión-exclusión educativa. Se presentan algunas características para entender la articulación de estas dimensiones y la forma en que cada una de ellas favorece $o$ dificulta la inclusión. De este modo, se muestra que, junto a los elementos estructurales y políticas educativas, la subjetivación del proceso en la vida cotidiana de la escuela garantiza mayores o menores niveles de inclusión. El ensayo defiende la noción de grupo para trabajar con una perspectiva más social de la educación y sus ventajas en el proceso de formación. Muestra cómo, en el proceso de inclusión-exclusión educativa, influyen de forma articulada las decisiones y las estrategias institucionales que organizan y estructuran la vida de los grupos escolares, sentando las pautas para la experiencia escolar.
\end{abstract}

Palabras clave: inclusión educativa, exclusión educativa, escuela.

\begin{abstract}
The school has the challenge of being the space where the direction of our societies is decided. In the pursuit of fairer and more inclusive societies, it is necessary to understand the socio-educational framework and its impact in a more complex way, especially the school group as a mediation space. For this purpose, a conceptual framework that includes inequalities from social / group subjectivity and places the gaze on institutional responsibility is proposed as a theoretical alternative for the analysis of educational inclusion-exclusion processes. The purpose of this essay is to discuss three dimensions of work necessary to guarantee inclusion mechanisms in educational institutions of secondary and higher education: the responsibility of school institutions in the guarantees for inclusion; the role of teachers in the design of school activity and the functioning of school groups from their relationship with the process of educational inclusion-exclusion. Some characteristics are presented to understand the articulation of these dimensions and the way in which each of them favors or hinders inclusion. In this way it is shown that, together with the structural elements and educational policies, the subjectification of the process in the daily life of the school guarantees higher or lower levels of inclusion. The essay defends the notion of a group to work with a more social perspective of education and its advantages in the training process.
\end{abstract}

Key words: educational inclusion, educational exclusion, schools

\footnotetext{
1 Profesora de la Universidad de La Habana en la Facultad de Psicología, Cuba. Psicóloga. Dirección electrónica: patricia.batista@psico.uh.cu

2 Profesor de la Universidad de La Habana en la Facultad de Psicología, Cuba. Psicólogo. Dirección electrónica: itorralbas@psico.uh.cu
}

Ensayo recibido: 6 de junio, 2018

Enviado a corrección: 12 de setiembre, 2018

Aprobado: 26 de noviembre, 2018 


\section{Introducción}

Las sociedades latinoamericanas se encuentran envueltas en un debate sobre su presente y futuro. Aunque con diferencias notables entre nuestros países, las transformaciones económicas y políticas imponen nuevos retos a la búsqueda y/o mantenimiento -en algunos casos- de la equidad, justicia e inclusión social. El escenario de la escuela no solo forma parte del contexto, sino que está - o debería estar- en el centro mismo de las reflexiones. En el empeño de una sociedad mejor, se debe mirar, cuidar y potenciar la escuela; proteger y ayudar al personal docente; fortalecer la buena institucionalidad y comprender de forma más compleja el entramado socioeducativo y su impacto.

Dentro de la escuela, el grupo escolar es "el lugar de mediación donde se da la relación entre la estructura social y la estructura individual, es un lugar de génesis y transformación de los sujetos y es un lugar de diagnóstico y operativo por excelencia" (Cucco, 1999, p.10). Por eso, puede ser tanto espacio de generación y movilización de la transformación como de reproducción de lo que puede dañar a una sociedad. Sin embargo, estos procesos no suelen ser objeto de análisis en profundidad. La mayoría de las diversas posturas teóricas y políticas tratan la escuela y los grupos solo como el escenario en el que ocurre el proceso de enseñanza aprendizaje. En muchos de esos casos, el interés instrumental no permite un análisis a profundidad de lo que la realidad grupal supone para los miembros y cómo esta impacta en el proceso educativo. Este es un modo de negar la fuerza de la subjetividad colectiva y también, de mantenerla silenciada y cómplice de los procesos de dominación.

Proponemos, entonces, una mirada a la escuela y al grupo educativo como realidades psicológicas. Ello supone visualizarlos como sujetos colectivos en los que emerge una subjetividad propia entendida como "construcción particular que se erige como producto de una permanente interpenetración de lo individual, lo grupal y lo social” (Fuentes, 2001, p. 28). Dicha subjetividad trasciende la individualidad de sus miembros, concretiza los modos y el hacer del contexto social en el que se encuentra. Es

Una permanente relación instituido-instituyente que desborda el marco grupal hasta llegar a las instituciones que los contienen donde simultánea y permanentemente se van reproduciendo mecanismos transformadores en la medida en que las fuerzas instituyentes se abren camino en las grietas de lo instituido. (Martín-Baró, 1989, p. 4) 
Son poco extendidos los constructos teóricos metodológicos con la complejidad suficiente para analizar de modo multideterminado la subjetividad grupal, así como su interdependencia con las producciones individual e institucional. De ese modo, se podrían comprender las articulaciones y condicionamientos de nuestros contextos sociales, políticos y económicos. Por tanto, se ha visto limitada la posibilidad de reconocer en el grupo al otro que nos contiene y nos introduce en el camino de hacernos sujetos sociales. Un proceso en el que "cada sujeto es asignado socialmente, es sujeto ideológico, y en la interacción grupal reproduce un modo de vida y reproduce lo sujetado en él; a su vez el espacio grupal es el lugar que permite recrear el modo de vida y recrear al sujeto" (Cucco y Losada, 1992, p. 05).

Proponemos como alternativa teórica partir de la mirada a los procesos de inclusiónexclusión educativa, con énfasis no en la dimensión individual -como suele trabajarse- sino como marco conceptual que comprende las desigualdades desde la subjetividad social/grupal y coloca la mirada sobre la responsabilidad institucional. El proceso de inclusión-exclusión educativa puede entenderse como un proceso o conjunto de procesos dinámicos, contextuales, multicausales, multidimensionales y de naturaleza relacional asociado a las potencialidades y dificultades de los sistemas educativos para garantizar equidad y participación plena de todo el estudiantado. (Ainscow, 2003; Echeita, 2008; Jiménez, Luengo y Taberner, 2009; Susinos y Parrilla, 2013). El abordaje de este fenómeno se convierte en una herramienta útil para "impactar en la transformación de los contenidos, enfoques, estructuras y estrategias que pueden garantizar una educación completa y de calidad a todos" (UNESCO, 2005).

Por eso, este ensayo presenta el análisis de tres dimensiones fundamentales: la responsabilidad de las instituciones escolares en las garantías para la inclusión; el papel del personal docente en el diseño de la actividad escolar, y el funcionamiento de los grupos escolares como posible contexto transformador. El debate propuesto sobre las posibilidades que brinda el grupo para comprender y transformar, en un sentido positivo el proceso de inclusión-exclusión educativa, según la necesaria articulación entre las tres dimensiones presentadas, se enriquece con conclusiones derivadas de investigaciones desarrolladas por ambos autores en al menos 30 grupos escolares de 15 instituciones docentes de enseñanza media y media superior en Cuba (Batista, 2018; Torralbas, 2017).

Para desarrollar el tema propuesto, el ensayo se estructurará en dos segmentos: el primero presenta la interdependencia institución educativa-docentes-grupo escolar, propia 
del proceso de inclusión-exclusión educativa; el segundo ofrece argumentos que amplían la discusión de acuerdo con algunos de los impactos posibles de estas dinámicas escolares, donde es posible encontrar evidencias de reproducción de las desigualdades, las cuales atentan contra el sostenimiento y ampliación de la inclusión. Corresponde a las ciencias sociales -y este ensayo es una contribución - pensar y accionar, desde estas herramientas para "desplazar el valor otorgado a la función instructiva de la escuela y resignificar su papel como agente de socialización" (Batista y Torralbas, 2017) para romper la dinámica de la dominación, crear y re-crear una praxis emancipadora.

\section{Las instituciones educativas, la clase y el grupo: Tres prioridades para la inclusión educativa}

Educar desde la perspectiva contemporánea es una acción absolutamente ligada a los contextos institucionales en los que se desarrolla. La escuela es el espacio por excelencia donde se jerarquiza la legitimidad y valor de los saberes, donde el estudiantado demuestra su capacidad para comprenderlos y ponerlos en práctica, lo que condiciona su incorporación al sistema laboral y social. Por tanto, es imprescindible comenzar preguntándonos sobre cómo los sistemas educativos son parte del proceso de inclusión-exclusión, qué elementos de su funcionamiento intervienen en el sostenimiento de garantías para la inclusión, cómo se relacionan con el papel del personal docente que diseña las clases, y al mismo tiempo, cómo influyen en el funcionamiento de sus grupos.

La inclusión educativa es solo posible cuando se ofrecen garantías institucionales al menos en tres direcciones: la presencia, el aprendizaje y la participación. La presencia es la posibilidad de asistir a las instituciones educativas; el aprendizaje con calidad es el modo en que los objetivos curriculares defienden la formación integral del estudiantado; la participación está asociada a la calidad de la experiencia educativa en el espacio docente, en la relación con los otros y en su percepción del proceso (Ainscow, 2003). La revisión de proyectos educativos en diversas partes del mundo muestra diferencias en el modo en que estas tres responsabilidades institucionales son asumidas.

Como generalidad prevalece un consenso sobre la prioridad de garantizar la presencia, pues se asume que ese es el primer paso para hablar de educación inclusiva, al menos en los niveles más básicos de formación. Las cifras actuales reconocen el aumento de las posibilidades de escolarización desde la enseñanza preescolar, con 93\% de matrícula en el 
nivel primario durante el período 2000-2015. La existencia de mayor disponibilidad de instituciones educativas en gran parte del mundo ha sido un logro, así como estrategias locales de obligatoriedad y gratuidad de la educación (UNESCO, 2015). Pudiéramos afirmar que el impacto de factores económicos y territoriales, así como diferenciadores en el acceso a la escuela ha disminuido. Sin embargo ¿ha sido suficiente?

La democratización del acceso escolar impone nuevos retos a los sistemas educativos. La apertura y gratuidad de las escuelas en muchos contextos han aumentado la diversidad de edades, género, orientación sexual, política, étnica, religiosa, cultural, etc. Malos manejos institucionales y estrategias docentes que no reconocen las potencialidades de lo diverso generan prácticas excluyentes sustentadas en prejuicios y estereotipos que favorecen la discriminación.

El aprendizaje y la participación parecieran estar atravesados por el diseño de estrategias educativas que muestran la dificultad de los diferentes actores vinculados al proceso docente de emplear herramientas para trabajar con lo diverso. Johnson (1972) advertía sobre la creciente tendencia de las instituciones a favorecer agrupamientos intraescolares con una composición intencionada:

Las variables más frecuentes que se han utilizado para construir los denominados agrupamientos homogéneos han sido la inteligencia, las capacidades académicas, el sexo, la religión, la raza y la edad (...) en algunos casos, son meros pretextos para realizar un tipo concreto de segregación cubierto con argumentos de rigurosidad científica (Aragonés, 1987, pp. 249-250)

Los agrupamientos homogéneos influyen de forma negativa en el proceso de inclusiónexclusión. La conformación de grupos muy diferentes entre ellos y homogéneos a su interior repercute, como mínimo, en las posibilidades de garantizar una experiencia educativa que favorezca la inclusión. Echeita (2013) comenta que "para progresar hacia planteamientos más inclusivos, los lugares son importantes, (...) resulta difícil aprender y reforzar determinadas competencias sociales, así como algunas actitudes y valores hacia la diversidad del alumnado (...) separando a los estudiantes" (p.105). Otras consecuencias pueden ser peligrosas polarizaciones, procesos de categorización exógena y endógena que ubiquen y mantengan a determinados grupos en posición de desventaja. 
Las escuelas de enseñanza media y media superior en Cuba muestran la tendencia a valorar, de modo desigual, a los grupos, unos denominados como "buenos" y diferentes de los "malos". Esta clasificación combina elementos propios del comportamiento y el rendimiento del estudiantado con algunas características sociodemográficas como el color de la piel, el acceso económico y el territorio de pertenencia (Batista, 2018; Batista y Torralbas, 2017).

Es necesario tener en cuenta esta tendencia pues los manejos institucionales impactan en el funcionamiento de los grupos cuando el estudiantado percibe que los grupos peor valorados son aquellos donde la mayoría pertenece a sectores sociales desfavorecidos por sus características sociodemográficas, evidenciando estereotipos y prejuicios que dificultan su inclusión. Agrupar a estudiantes por las similitudes de rendimiento docente y comportamiento limita sus posibilidades de desarrollo, derivado de la interinfluencia propia de los funcionamientos grupales. Las nominaciones y consecuentes expectativas cuando son conocidas por el estudiantado suelen ser incorporadas a la construcción de su autoimagen, reforzando el desbalance entre sus potencialidades y debilidades.

Los grupos pueden reproducir las expectativas que sobre ellos se tiene. Así, los "buenos" son altamente competitivos, confieren mucha importancia al rendimiento académico y lo reconocen como su principal criterio de diferenciación. Expresan malestar debido a dificultades en sus relaciones interpersonales, las cuales no son advertidas ni manejadas por el cuerpo docente. En muchos casos, se constata dificultad en la coordinación entre los miembros del grupo para desempeñarse en algunas tareas institucionales como la organización de los horarios, la realización de trabajos prácticos y la planificación de actividades (Batista, 2018; Batista y Torralbas, 2017).

Los grupos "malos" se identifican con comportamientos como la falta de interés por el estudio, la indisciplina y la ausencia a clase. Dichas características son asumidas como parte del proceso de diferenciación del resto de la escuela y suelen ser bien valoradas ante la necesidad de garantizar una autoimagen positiva. Se confiere más importancia a garantizar buenas relaciones interpersonales entre sus miembros. Como consecuencia se reproducen comportamientos que atentan contra la tarea del grupo - "fugarse" de clases, sabotear la actividad docente, usar incorrectamente el uniforme, cometer indisciplinas fuera y dentro del aula - que no se corresponden con los objetivos institucionales (Batista, 2018; Batista y Torralbas, 2017). 
Esta tendencia profundiza un efecto negativo de las diferencias que se traducen en desigualdades, sobre todo si las valoraciones traen aparejadas la asignación de ventajas y desventajas. Es común la asignación de profesores y profesoras con más experiencia a los grupos "buenos", así como territorios con mejores condiciones. Cuando nos referimos al territorio tenemos en cuenta la reconocida la influencia de las condiciones del medio físico, por ejemplo: el tamaño de la institución, el diseño físico y los niveles socioeconómicos en el modo en que acontece el proceso docente educativo (Newman citado por Sangrador, 1987). Algunos estudios (Wollin y Montage; Sommer y Olsen) han mostrado la influencia de la comodidad y flexibilidad de los espacios educativos en la relación docente-estudiante, así como la participación y mejor comunicación por parte del estudiantado (Sangrador, 1987).

Por tanto, cuando las instituciones educativas asignan locales de trabajo y recursos que ratifican la valoración desigual que se tiene de sus diferentes grupos agudizan las diferencias y sus consecuencias negativas y estigmatizantes. Las condiciones constructivas favorables, la amplitud y mejor visibilidad de las aulas asignadas a los grupos "buenos", acompañadas de un mejor mobiliario, medios de enseñanza y equipos de iluminación y ventilación favorecen una mejor experiencia educativa que motiva de forma positiva al aprendizaje. Los territorios con malas condiciones empobrecen la vida del grupo y limitan su actividad, constituyen un complemento al clima de indisciplina y resistencia a la actividad de estudio, asimismo, refuerzan su autoimagen deteriorada. La asignación de territorios y de materiales con diferencia de calidad marcada es un proceso institucional inconsciente, en el que se reproduce lo que sucede a escala macrosocial. No obstante, pocas veces existe una reflexión-acción profunda sobre estos puntos.

La posición que asume la escuela frente al reto de la inclusión educativa se constata en los modos en que el profesorado juega su papel frente a los grupos escolares, en otras palabras, las estrategias institucionales se concretan y explicitan en los diseños de las clases que favorecen o no la inclusión. Cuando la institución educativa organiza de forma homogénea a sus grupos y ofrece clasificaciones como las mencionadas anteriormente, que resaltan el buen de desempeño de un grupo en detrimento de otro, introduce sesgos importantes. Como consecuencia de las expectativas generadas, el claustro docente comienza a incorporar y transmitir prácticas hacia sus estudiantes que consolidan las desigualdades. Por ejemplo, es más probable que el profesorado se muestre agresivo y descontento hacia estudiantes de grupos "malos", en los que se estructuren clases menos 
participativas y creativas, regañando de forma más violenta o utilizando la evaluación como método para garantizar la disciplina, incluso, es posible que aun cuando se diseñen estrategias de trabajo se mantenga un trato prejuiciado hacia sus posibilidades de transformación (Batista, 2018; Batista y Torralbas, 2017).

Es necesario lograr una correcta articulación entre el diseño curricular y la práctica educativa, cuando ambos muestran una atención desmesurada a la transmisión de contenidos académicos que no garantizan la formación integral del estudiantado como ciudadanos, las estrategias para la inclusión se ven limitadas. El currículo adquiere más relevancia y pertinencia en la medida que sus principios, concepciones psicopedagógicas, metodológicas, filosóficas y sociológicas se correspondan con la expectativa social, así como con la formación integral que deben alcanzar los grupos estudiantiles en correspondencia con los escenarios socioeconómicos, políticos y culturales y aquellos valores nacionales y universales para ejercer su función social como ciudadano (Gayle y Leal, 2011, p.11).

En Cuba, la educación se encuentra en medio de su Tercera Etapa de Perfeccionamiento, donde la adaptación curricular es una de sus premisas esenciales a partir del reconocimiento de las limitaciones que presentan los planes de estudio para complementar una formación integral de sus estudiantes. Las nuevas propuestas de transformación proponen flexibilizar el diseño curricular manteniendo uno general que homogenice la formación dentro de un mismo nivel de enseñanza, pero con la posibilidad de complementarlo con uno propio, generado por cada centro docente de acuerdo con sus peculiaridades.

Esta posibilidad abre nuevos caminos, sobre todo para el trabajo con las problemáticas que emergen en los grupos escolares, en especial la inclusión educativa. Sin embargo, también supone grandes retos. Las escuelas y las personas docentes deben estar preparadas para entender las particularidades de su contexto escolar y proponer alternativas educativas que sean coherentes. Sin la adecuada preparación puede continuar en la práctica un proceso educativo esencialmente academicista, poco atento al mejor desenvolvimiento de las relaciones sociales. Este reto es aún mayor en la enseñanza media superior, donde el diseño curricular tradicional ha puesto el énfasis en la "trasmisión" de conocimientos académicos. La formación de valores, la preparación socioemocional del estudiantado y la educación de habilidades que favorezcan las relaciones sociales están carentes en la exposición de contenidos y ejercicios. Esta realidad se expresa en el pobre trabajo realizado 
con los textos y temas emergentes durante la clase, así como en la indiferencia hacia las problemáticas grupales evidentes (Batista, 2018).

En otros casos, fundamentalmente en enseñanza media, se le confiere mayor importancia al funcionamiento grupal. Sin embargo, no siempre son trabajados de forma favorable. Los y las docentes pueden planificar acciones que enriquezcan la vida de los grupos, pero generalmente son diseñadas al margen de su tarea primaria, y por tanto limitan la repercusión posible en la organización grupal. Son frecuentes las actividades extraescolares como cumpleaños colectivos, excursiones y actividades deportivas fuera de la escuela.

Se trazan estrategias de aprendizaje cooperativo para favorecen el desempeño docente de algunos estudiantes a partir de dos modalidades: las "casas de estudio" y el "apadrinamiento". Cada una de ellas tiene potencialidades y limitaciones. Las "casas de estudio" favorecen los sistemas de apoyo generados por el propio grupo para complementar las herramientas que brinda la escuela. Su conformación suele ser intencionada por la persona docente para evitar que los agrupamientos sean por afinidad, pese a ello, en la práctica suelen reunirse de acuerdo con los propios subgrupos y presenta las mismas dificultades de integración que se evidencian en el aula. Por su parte, el "apadrinamiento" consiste en la conformación de dúos de trabajo donde un estudiante de buen desempeño docente se responsabiliza de apoyar a otro que presenta dificultades. La práctica muestra que la asignación de ambos roles influye en la autoimagen de los implicados y su papel frente al grupo. Además, jerarquiza las relaciones asignando más poder a quienes tienen los mejores resultados, y provoca conflictos interpersonales poco atendidos por el personal docente.

Es un verdadero peligro para garantizar la inclusión que el claustro docente tenga poco conocimiento sobre la vida del grupo y sus miembros, o de las herramientas para trabajar con él, pues restringe la planificación de estrategias que permitan un clima favorable. En la mayoría de los casos, estas limitaciones están estrechamente vinculadas con la carencia de recursos pedagógicos por parte de los y las docentes, dificultades que se derivan esencialmente de su formación general y postgraduada.

Afirmamos que en una escuela se trabaja para garantizar la inclusión educativa cuando, de forma consciente y planificada, se impulsan estrategias para asumir la diversidad escolar como una potencialidad educativa. En las aulas pueden converger estudiantes de 
diferente color de piel, sexo, de diversa orientación sexual, religión, consumo cultural, procedencia económica y territorial, capacidades físicas o habilidades cognoscitivas y emocionales. Dichas diferencias pueden dialogar en un espacio abierto para la comunicación y el intercambio, donde la presencia del otro no suponga reproducción de estereotipos y prejuicios asociados a desigualdades que tienen lugar en el contexto social. Es el personal docente el principal encargado de hacer efectivas dichas estrategias incorporándolas a su práctica cotidiana en el aula.

La inclusión-exclusión educativa es, en esencia, un proceso relacional que trasciende la subjetividad individual, por tanto, el funcionamiento del grupo escolar es un espacio propicio para entender los modos en que está ocurriendo esa relación escueladocentes/diseño de la clase. Es la oportunidad de comprender problemáticas específicas del contexto escolar, como la violencia y la discriminación entre compañeros de estudio, no solo como parte de dinámicas interindividuales, sino también desde la responsabilidad compartida por el sistema educativo y su profesorado. Es necesaria una mirada atenta al grupo, entendiéndolo en el contexto del cual es parte, como primer paso en la búsqueda de alternativas de estudio e intervención.

\section{Mirando los grupos escolares: La reproducción de la desigualdad social a través de su funcionamiento.}

Los estudios en las escuelas sobre violencia, bullying, acoso, fracaso escolar, etc., tienen dos peligros fundamentales: en primer lugar, reducir estos procesos a vínculos interindividuales. Nuestra visión apuesta por entender que la naturaleza de estas relaciones es producción grupal y, a su vez, condicionamiento social. En segundo lugar, y más peligroso aún, es reducir los complejos procesos de reproducción de la desigualdad a categorías que describan comportamientos específicos. Ello hace que se pierda la razón última de la producción de ese tipo de conductas.

La mirada de la que parten nuestras reflexiones hacia los grupos permite una lectura abarcadora sobre la influencia de factores macro estructurales e individuales que se concretizan en el espacio del aula. El reconocimiento de una subjetividad grupal y el papel protagónico de las instituciones en dichos procesos son parte de nuestras premisas. Dicho en palabras de Mara Fuentes (2001): 
Los grupos no son lo grupal. Por eso, (...) se hace necesario diferenciar la comprensión de los agrupamientos humanos como contextos de una comprensión psicosocial de los mismos en tanto espacios psicológicos de construcción y expresión de la subjetividad, tratando de diferenciar un enfoque de trabajo tradicional "en el grupo" de un enfoque "desde el grupo". Esta comprensión nos permitirá desarrollar un dispositivo interventivo que permita una mirada de "lo grupal" y una utilización efectiva del grupo en el proceso de cambio que se pretende. (p. 29)

Analizar el grupo educativo desde la perspectiva de la psicología social nos permite analizar múltiples aristas que pueden ayudar a optimizar el proceso educativo. El modo de entender el vínculo y su importancia es reconocer en el grupo al otro que nos contiene y nos introduce en el camino de hacernos sujetos sociales (Cucco y Losada, 1992). A continuación, presentaremos algunos indicadores a emplear en esta mirada.

Los procesos de diferenciación a lo interno de los grupos son consustanciales a su existencia. Por tanto, la mera existencia de fronteras a su interno no constituye indicador de mayor o menor inclusión. Sin embargo, sus componentes y la naturaleza de las relaciones entre dichos subgrupos pueden ser utilizadas para realizar un análisis de los procesos de diferenciación y comparación que devienen en el establecimiento de jerarquías, otorgamiento de ventajas y desventajas, además de conflictos. En este tipo de análisis sugerimos el uso de técnicas asociativas que brindan un material muy revelador, pues estas superan las resistencias de los miembros del grupo. Resulta vital explorar si el propio grupo identifica relaciones de conflicto entre los miembros y si estos obstaculizan la comunicación y profundizan las distancias entre cada uno de los subgrupos. También, cuánto entorpecen la propia actividad de estudio, pues suponen dificultad en encontrar consenso ante decisiones relacionadas con la organización del horario docente o la realización de trabajos en equipo, entre otros.

El contenido de las etiquetas que utilizan las personas integrantes del claustro para nombrar a cada subgrupo permite comprender las razones que diferencian estas agrupaciones entre sí. En sí mismas algunas nominaciones son evidencias de agresiones verbales hacia los exogrupos o identificaciones que satisfacen la autoestima positiva del endogrupo. En escuelas de enseñanza media y media superior en Cuba, es frecuente encontrar subgrupos que son valorados negativamente de acuerdo con sus características 
sociales, por lo cual reciben etiquetas, tales como como "los/as fiñes", "las tímidas", "los/as mongos/as", "los pencos", "los bobos", "los anormales". Suelen ser estudiantes con dificultades para las relaciones sociales, que no se integran a las actividades que realizan la mayor parte del grupo y aquellos de mayor estatus. Estos subgrupos reciben burlas y son aislados por sus compañeros. Otras denominaciones como "los/as feos/as", "los churrosos" y "los gordos" muestran la importancia de la apariencia física en estas edades, sobre todo en enseñanza media (Batista, 2018, Torralbas, 2017).

La valoración negativa también incluye a otros pequeños grupos de estudiantes, que se destacan por su mal comportamiento en el aula y reciben el rechazo de una parte del grupo. Llamados "los indeseables", "los insoportables" o "los problemáticos"3 se caracterizan por pertenecer al sexo masculino y ser más criticados por las mujeres. Su condición suele ser respaldada por el personal docente quienes refuerzan dichas categorías. También se distinguen subgrupos de muchachas mal valoradas por cualidades personales asociadas a la tenencia de recursos económicos que las diferencian del resto: “las plásticas", "las pijas”, "las cuquis"4, reciben agresiones verbales más sutiles, pero son más criticadas por otras mujeres con menos ingresos económicos y/o consumos culturales más modestos. Suelen ser subgrupos más pequeños y cerrados.

En contraposición aparecen subgrupos generalmente más numerosos y mejor valorados por sus características, las cuales favorecen las relaciones sociales: "divertidas/divertidos", "populares", "las loquitas", "los cómicos", “las tremendas", "los gozones" ${ }^{5}$. Estos son reconocidos por favorecer un clima positivo dentro del aula, aunque suponga indisciplinas leves. En ocasiones son los subgrupos que más se contraponen a los mencionados anteriormente.

El consumo cultural (fundamentalmente musical), el uso del tiempo libre y los lugares que frecuentan suelen establecer fronteras dentro del grupo. Estas diferenciaciones tienen menos conflictos asociados, excepto en los casos en que supone una clara distinción en cuanto al nivel económico de sus miembros, por ejemplo, "los Gamers" 6 , "los frickies" y "los

\footnotetext{
${ }^{3}$ Estudiantes que se destacan por su mal comportamiento dentro del aula. Generalmente alteran la disciplina 0 atacan verbalmente a sus compañeros y compañeras. resulta común que pertenezcan al sexo masculino.

${ }^{4}$ Estudiantes que se destacan por el uso de accesorios, cuidado del cabello y maquillaje, los cuales suponen altos ingresos económicos. Generalmente pertenecen al sexo femenino.

5 Estudiantes que mantienen un clima positivo en el grupo, cometen indisciplinas leves pero sostienen buenas relaciones con la mayor parte del grupo.

${ }^{6}$ Estudiantes que invierten gran parte de su tiempo libre haciendo uso de las nuevas tecnologías, en especial, los videojuegos.
} 
que se visten bien", tienen mejor valoración y pueden entrar en contradicción con "los deportistas", "los reparteros" ${ }^{8}$ que generalmente engloba a estudiantes de más bajos ingresos.

En relación con el desempeño académico hay distinciones entre "los/as brutos/as" y "los/as inteligentes" o "los/as estudiosos/as". Es uno de los criterios que más conflictos genera, y cuyas fronteras están más claras, sobre todo en los grupos "buenos" dentro de cada escuela. Es secundado por las acciones del claustro que refuerzan dichas etiquetas. Aquellos que reciben peor valoración ("los brutos", "los sin cerebro") corresponden a denominaciones negativas y frecuentes muestras de rechazo: "las puntualitas", "creyentes", "puchis".

La conformación de subgrupos dentro del aula influye en el aprovechamiento del proceso docente educativo, establece criterios de pertenencia que predisponen el comportamiento de sus miembros en cuanto a variables como sexo, color de piel, nivel económico, procedencia social, entre otros. El estudiantado reconoce que las relaciones entre los subgrupos se ven afectadas por sus diferencias y que como consecuencia ocurren algunos conflictos, fundamentalmente agresiones verbales. La mayoría de sus causas y manifestaciones son naturalizadas por considerarlas normales, dado las características de la edad y el contexto en que viven. Esa naturalización contiene el peligro mayor de la reproducción social: "siempre es o ha sido así", "es normal”, "así es la vida", son expresiones anti transformadoras que expresan la reproducción de un orden social favorecedor de unos pocos.

Para las y los estudiantes está muy clara la existencia de fronteras a lo interno de sus grupos. Reconocen también las jerarquías y expresiones de conflicto, pero en este caso son experimentadas con malestar por afectar las relaciones entre compañeros y compañeras, y suponer desventajas para algunos sectores del aula. El profesorado en ocasiones advierte la existencia de subgrupos y reconoce que las divisiones están dadas por diferencias en el rendimiento académico, ingresos económicos, gustos, intereses futuros, sexo o formas de pensar del estudiantado. Sin embargo, no tienen las herramientas suficientes para incidir sobre la dinámica de los grupos y ofrecer alternativas de transformación.

\footnotetext{
${ }^{7}$ Estudiantes que por su forma de vestir y actuar aparentan estar asociados al consumo de Rock and Roll.

8 Estudiantes que por su forma de vestir y actuar aparentan estar asociados al consumo de ritmos populares como el Reguetón.

${ }^{9}$ Estudiantes que se destacan por su buena disciplina y rendimiento docente pero no son bien valorados por sus compañeros por atender más la actividad de estudio que las relaciones sociales.
} 
La socialización en el ámbito escolar es fundamental, la interacción en el grupo escolar prepara para vivir en sociedad. A lo interno de los grupos escolares se vive un ámbito, un recorte de la sociedad en la que la escuela está insertada. Se comienza, de forma espontánea y condicionado por la actividad docente y el rol del claustro, a conformar una estructura grupal - asignación y asunción de roles, status, territorios- que comportan elementos de reproducción de las desigualdades. Una expresión son estudiantes que comienzan a ser preferidos, rechazados, aislados o controvertidos. El análisis de los tipos sociométricos y de su connotación es una alternativa para entender grupos escolares y conducirlos de modos más innovadores. La definición de los criterios de éxito es vital para comprender los ejes estructurantes de la vida de los grupos, lo que se traduce en herramientas para transformar ejes que suponen la reproducción de perfiles de ventaja social y re-construir oportunidades y alternativas.

Las investigaciones que sustentan estas reflexiones muestran cómo en grupos escolares de adolescentes en Cuba, los preferidos en los grupos son personas de color de piel blanca, elevado rendimiento académico y procedente de familias con un nivel educativo alto. Estas personas son más respaldadas por sus docentes, quienes se muestran tolerantes ante sus indisciplinas o dificultades académicas. Además, juegan un papel importante en la toma de decisiones dentro del grupo. Por su parte, los rechazados, por lo general, son estudiantes negros y/o mestizos. Ellos son los que más malestar manifiestan con el contexto educativo. En su base podríamos hipotetizar la presencia de prejuicios raciales. Su estatus dentro del grupo los coloca en el centro de agresiones verbales, las cuales suelen devolver de lo mismo, lo que complejiza las relaciones dentro del aula. Esta condición, en algunos casos, es respaldada por sus docentes quienes pueden justificar algunos comportamientos discriminatorios (Batista, 2018; Torralbas, 2017).

Aunque ya estos elementos son sólidos, la realidad es más compleja aún. Son tan fuertes los aprendizajes sociales que hemos constatado, con herramientas provenientes de la Teoría de las Redes Sociales, que incluso cuando tenemos adolescentes de color de piel negra con un estatus de preferido, tienen redes de conexión con su grupo menos extensas y más débiles. De igual modo, los adolescentes de piel blanca con estatus aislado, tienen redes más amplias y más sólidas que otros en su misma condición.

Es por ello que afirmamos que necesitamos agudizar la mirada y establecer nuevos indicadores para comprender cómo la reproducción de los perfiles de desventaja ocurre no 
solo delante de la mirada de las instituciones escolares, sino con su aprobación. No hacer nada, también es un modo de hacer.

Del mismo modo, se observa un movimiento en el que las relaciones de género se modifican. Mientras en las etapas iniciales de la adolescencia las mujeres son más preferidas y prosociales que los hombres, al final de esa edad y comienzo de la juventud los hombres se vuelven más centrales en la vida del grupo, llegando a ser más preferidos que las mujeres en los aspectos de relaciones sociales. En un estudio de Plazas et al. (2009), se constataron resultados similares cuando comparaban la enseñanza media con la superior.

El funcionamiento de los grupos escolares muestra dificultades para sostener la inclusión educativa. Las diferencias constituyen elementos que colocan en desventaja a parte del estudiantado y se hace evidente en los calificativos con los que se nombran y la forma en que se configuran jerárquicamente sus relaciones. Lo anterior es el resultado del impacto de prácticas docentes excesivamente centradas en el desarrollo de habilidades académicas y con poco énfasis en sostener la inclusión. El personal docente debe tener herramientas para entender cómo ocurren dichos procesos en su grupo, y desde ahí planificar acciones transformadoras. La concienciación sobre la importancia de estas transformaciones es solo posible si es una propuesta de los sistemas educativos y las estrategias que las escuelas diseñan de forma consciente y planificada en pos de la inclusión.

\section{Reflexiones finales}

Garantizar la inclusión educativa debe ser tarea prioritaria en las instituciones escolares. Se debe responder con efectividad a la creciente demanda de la construcción de una mejor sociedad, donde, en principio, sus ciudadanos deben estar preparados para convivir en un mundo diverso. No hay sociedad libre, justa y emancipada sin justicia y democracia en la educación. Así lo entendió José Martí. La atención a procesos subjetivos en relación con elementos estructurales es vital. En las instituciones escolares es necesario revisar dicha dimensión estructural, la cual ha naturalizado prácticas en esencia excluyentes, que jerarquiza y otorga ventajas y desventajas educativas a los diferentes sectores sociales que en ella comparten. Solapar los procesos subjetivos que tienen lugar en las prácticas docentes invisibiliza la peligrosa reproducción de procesos macrosociales con una carga desintegradora. 
El ensayo ha mostrado que en el proceso de inclusión-exclusión educativa influyen de forma articulada las decisiones y las estrategias institucionales que organizan y estructuran la vida de los grupos escolares, lo que sienta las pautas para la experiencia escolar. En ese contexto institucional la práctica educativa es encabezada por el claustro docente, que juega un papel esencial en la garantía de la inclusión pues explicitan en su diseño de clase, los elementos que el sistema educativo organiza. Sin embargo, con los elementos presentados, este ensayo propone entender la relación que existe entre ambos agentes y el grupo escolar con el proceso de inclusión-exclusión educativa junto a los elementos estructurales (garantías de acceso, legislaciones, reglamentos escolares, políticas educativas, entre otros), la subjetivación del proceso en la vida cotidiana de la escuela y sus dinámicas es la que garantiza mayores niveles de inclusión.

Mientras las alternativas para sostener la inclusión partan de estrategias de trabajo individual, las posibilidades de acción se verán limitadas. Las estrategias puramente individuales contradicen la preparación de los sujetos para la vida en colectivo, declarada como objetivo transversal y coherente con el sistema social que se promueve. Además, dichas estrategias se limitan a la compensación, y en la práctica no suplen las demandas para la educación inclusiva. En cambio, la noción de grupo y las ventajas que supone de cara al proceso de formación resultan más factibles. Trabajar con una perspectiva más grupal, trabajar con una perspectiva más social de la educación es:

contribuir a perfeccionar los mecanismos a través de los cuales se favorece la relación de correspondencia entre el proyecto social y el proyecto individual ya que (...) no hay proyecto social que pueda ser alcanzado, ni llevado a vías de éxito si las personas, que son los encargados de ejecutarlos, no lo incorporan, en diferentes medidas, a sus proyectos individuales de vida. (Fuentes, 2001, p. 28)

Este no es asunto de una sola ciencia, ni siquiera es asunto solo de los científicos sociales. Es asunto de toda una sociedad que define su destino en sus grupos escolares. En general, personas directivas, docentes y ciudadanas, deberían trabajar por concienciar los sesgos estructurales que median sus relaciones sociales cargadas de una producción subjetiva que desafortunadamente reproduce desigualdades. Es necesario tener en cuenta la reproducción solapada de la estigmatización presente en prácticas como la nominación desigual de los grupos escolares por parte del profesorado o el establecimiento de etiquetas 
que develan relaciones asimétricas entre estudiantes. También, se debe considerar la socialización de la desigualdad y la normalización de la exclusión emergentes en un contexto educativo que presenta dificultades para reconocer las potencialidades de la diversidad para una formación más integral. Finalmente, la emancipación liberadora, la felicidad de todas y todos en espacios educativos diversos requiere reflexiones inmediatas, aún más, requiere acciones de transformación.

\section{Referencias}

Ainscow, Mel. (2003). Desarrollo de Sistemas Educativos Inclusivos. San Sebastián: The University of Manchester.

Aragonés, Juan. (1987). Los grupos de iguales en la escuela. En Carmen Huici, Estructura y Procesos de Grupo II (pp. 214-262). Madrid: UNED.

Batista, Patricia y Torralbas, Jorge (2017) Inclusión Educativa: retos para la Enseñanza Media Superior en Cuba. Temas, (89-90), 97-103.

Batista, Patricia. (2018) Caracterización del proceso de inclusión-exclusión educativa en instituciones docentes de La Habana (Tesis en opción al grado académico de Máster en Psicología Educativa). Facultad de Psicología, Universidad de La Habana. La Habana, Cuba.

Cucco, Mirtha. (1999). Los procesos grupales, base del aprendizaje. En Atención a la Diversidad en E.S.O. Actualización Científico-Didáctica (pp.9-20). Aranjuez: Centro de profesores y recursos de Aranjuez.

Cucco, Mirtha, y Losada, Luis. (1992). El espacio grupal. Espacio de génesis y transformación. IV Encuentro Latinoamericano de psicoanalistas y psicólogos marxistas. La Habana.

Echeita, Gerardo. (2008). Inclusión y Exclusión educativa. De nuevo "Voz y Quebranto". Revista Electrónica sobre Innovación y Cambio en Educación, 1-29.

Echeita, Gerardo. (2013). Inclusión y Exclusión educativa. De nuevo "Voz y Quebranto". Revista Iberoamericana sobre Calidad, Eficacia y Cambio en Educación, 11(2), 99-118.

Fuentes, Mara. (2001). ¿Por qué el grupo en la Psicología social? Revista Cubana de Psicología, 18(1), 28-33.

Gayle, Arturo y Leal, Haydeé. (2011). El currículo de la escuela cubana actual. El tratamiento a la diversidad mediante estrategias educativas. La Habana, Cuba: Sello editor Educación Cubana. 
Jiménez, Magdalena; Luengo, Julián y Taberner, José. (2009). Exclusión social y Exclusión educativa como fracasos. Conceptos y líneas para su comprensión e investigación. Profesorado. Revista de Currículum y Formación de Profesorado, 13(3), 11-49.

Martín-Baró, Ignacio. (1989). Sistema, grupo y poder. Psicología Social desde Centroamérica. San salvador: UCA.

Plazas, Elberto Antonio; Morón, María Lourdes; Santiago, Antonia; Sarmiento, Humberto; Ariza, Sara Elvira y Patiño, Carlos Darío (2010). Relaciones entre iguales, conducta prosocial y género desde la educación primaria hasta la universitaria en Colombia. Universitas Psychologica, 9(2), 357-369.

Sangrador, José. (1987). El ambiente físico en los grupos. En Carmen Huici, Estructura y Procesos de Grupo I (pp. 331-358). Madrid: UNED.

Susinos, Teresa y Parrilla, Ángeles. (2013). Investigación Inclusiva en tiempos difíciles. Certezas provicionalesprovisionales y debates pendientes. Revista Iberoamericana sobre Calidad, Eficacia y Cambio en Educación, 11(2), 88-98.

Torralbas, Jorge. (2017) Diagnóstico del proceso de cohesión grupal en contextos escolares (Tesis en opción al grado académico de Máster en Psicología Educativa). Facultad de Psicología, Universidad de La Habana. La Habana, Cuba.

UNESCO. (2005). Informe de Seguimiento de la EPT en el Mundo. La Educación para Todos: el imperativo de la calidad. Paris: Ediciones UNESCO. 